\title{
Saúde e ambiente na Amazônia brasileira
}

\section{Health and environment in the Brazilian Amazon}

Rosa Carmina de Sena Couto - Doutora em Saúde Pública, pela Escola Nacional de Saúde Pública/FIOCRUZ. Professora Associada IV da Faculdade de Medicina da UFPA (aposentada).E-mail: rosasenacouto@gmail.com

\section{Resumo}

$\mathrm{O}$ artigo analisa aspectos relacionados aos problemas ambientais globais: mudança climática e perda da biodiversidade no contexto do desmatamento e da saúde na Amazônia brasileira. Ressaltamos que a Amazônia é o epicentro da crise da biodiversidade e que o encerramento do desmatamento é necessário para diminuir as emissões brasileiras de gases de efeito estufa. Com os objetivos propostos, fundamentamos nossa análise no debate recente sobre a questão. O Sistema Único de Saúde (SUS) foi problematizado no sentido de ampliar suas abordagens diante dos novos desafios para enfrentar as demandas ambientais para a saúde humana considerando as especificidades regionais: o fator amazônico, a diversidade étnica, características do território, regiões de saúde e novas abordagens. Concluímos sinalizando para o encerramento do desmatamentonaAmazôniaeapromulgação de um novo modelo de desenvolvimento bioeconômico, conciliando com os recursos da biodiversidade da floresta e os saberes ancestrais favorecendo as populações locais.

\begin{abstract}
This article analyzes aspects related to global environmental problems: climate change and loss of biodiversity in the context of deforestation and health in the Brazilian Amazon. We emphasize that the Amazon is the epicenter of the biodiversity crisis and that the end of deforestation is necessary to reduce Brazilian emissions of greenhouse gases. With the proposed objectives, we base our analysis on the recent debates on the issue. The Unified Health System (SUS) was discussed in order to broaden its approaches in the face of new challenges to face environmental demands for human health considering regional singularities: the Amazonian factor, ethnic diversity, characteristics of the territory, health regions and new approaches. We concluded by signaling the necessity to end deforestation in the Amazon and the enactment of a new pattern of bioeconomic development, reconciling with the resources of the forest biodiversity and ancestral knowledge favoring local populations.
\end{abstract}

\section{Palavra-chave}

\section{Keywords}

Mudança Climática. Biodiversidade. Desmatamento. Saúde. Amazônia. SUS.

Climate change. Biodiversity. Deforestation. Health. Amazon. Unified Health System (SUS). 


\section{INTRODUÇÃO}

É inquestionável a relação saúde e ambiente. A relação sociedade-natureza determina o padrão de exploração dos recursos naturais e o padrão de consumo da sociedade, determinando saúde, qualidade de vida e bem-estar, ou determinando escassez, desigualdade e doenças (COUTO, 2018).

As atividades antrópicas degradam os ecossistemas e desestruturam as bases ecológicas do planeta. Os seres humanos dependem essencialmente dos ecossistemas e dos recursos naturais, bens e serviços que eles podem fornecer: alimentos, água limpa, controle de doenças, regulação do clima, satisfação espiritual e estética. Os ecossistemas são a base da vida e são responsáveis pela segurança alimentar, hídrica e climática do planeta. A saúde humana depende da saúde ambiental (MILLENNIUM ECOSYSTEM ASSESSMENT, 2005).

A Amazônia, por ser uma região megabiodiversa, é submetida à exploração predatória de seus recursos naturais e está implicada com os problemas ambientais globais: mudança climática e perda da biodiversidade, assim como os impactos na saúde da população humana decorrentes de projetos de desenvolvimento (hidrelétrica, agricultura, mineração e ferrovias, rodovias e portos), os quais pressionam o Sistema Único de Saúde (SUS) que, na maioria das vezes, não consegue atender adequadamente essas demandas (COUTO, 2019).

O objetivo deste trabalho é problematizar alguns aspectos relacionados aos problemas ambientais globais: mudança climática e perda da biodiversidade no contexto do desmatamento e da saúde na Amazônia brasileira.

\section{PROBLEMAS AMBIENTAIS GLOBAIS}

A Organização das Nações Unidas (ONU) alerta que o planeta se dirige para o colapso climático, sanitário e social. Ela ressalta que ainda há tempo de reverter esse quadro, reduzindo as emissões de gases de efeito estufa que provocam as mudanças climáticas, diminuindo os níveis de consumo e protegendo a água e a biodiversidade. $\mathrm{O}$ estilo de vida com base no consumo de combustíveis fósseis (gás, petróleo, carvão) levou a humanidade para o maior desafio global, que são as mudanças climáticas (ARANDA, 2019).

É consenso que a queima de combustíveis fósseis (gás, petróleo e carvão) contribuam para o aquecimento do planeta, assim como o desmatamento das florestas tropicais. Essas atividades liberam gases de efeito estufa: dióxido de carbono $\left(\mathrm{CO}_{2}\right)$, metano $\left(\mathrm{CH}_{4}\right)$, óxido nitroso $\left(\mathrm{N}_{2} \mathrm{O}\right)$ e outros (ORGANIZAÇÃO MUNDIAL DA SAÚDE, 2009). 
Em 2015, foi realizada em Paris a 21ª Conferência das Nações Unidas sobre as Mudanças Climáticas (COP21), com o objetivo de celebrar um novo acordo sobre o clima que respondesse globalmente à ameaça da mudança climática, além de reforçar a capacidade dos países para lidar com os efeitos desse problema. O acordo foi aprovado por 195 países representados na conferência (BRASIL, 2015).

Assim, o Acordo de Paris enfatiza a necessidade de "[...] manter o aumento da temperatura média global a menos de $2^{\circ} \mathrm{C}$ acima dos níveis industriais e promover esforços para limitar o aumento da temperatura a $1,5^{\circ} \mathrm{C}$ acima dos níveis pré-industriais" (ONU, 2015, p. 2).

O Brasil, presente na conferência, referendou o Acordo de Paris e se comprometeu em reduzir suas emissões de gases de efeito estufa em: 37\% abaixo dos níveis de 2005, em 2025; e em 43\% abaixo de 2005, em 2030. O país também se comprometeu em restaurar 12 milhões de hectares de florestas até 2030, além de alcançar $45 \%$ de energias renováveis na composição da matriz energética em 2030, entre outros compromissos assumidos (BRASIL, 2015).

O último relatório do Painel Intergovernamental sobre Mudanças Climáticas (IPCC, sigla em inglês), órgão ligado à Organização das Nações Unidas (ONU), analisa as perspectivas de limitar o aquecimento global em até $1,5^{\circ} \mathrm{C} \mathrm{em}$ relação ao período pré-industrial e indica que são necessárias mudanças urgentes na forma como usamos a terra, como geramos energia, como nos movemos, como produzimos e moramos (IPCC, 2018).

De acordo com o IPCC, se a emissão de gases de efeito estufa continuar a subir na taxa atual, o mundo estará $1,5^{\circ} \mathrm{C}$ mais quente entre 2030 e 2052, em comparação à temperatura registrada antes da Revolução Industrial. Esse aumento de temperatura é suficiente para bagunçar o sistema climático global e provocar efeitos perigosos, como tempestades mais fortes e mais frequentes, períodos mais longos e mais intensos de seca, mais derretimento do gelo nos polos, entre outros (IPAM, 2018, não paginado).

Nesse contexto, o IPAM ressalta que as florestas atualmente removem cerca de $1 / 4$ de CO2 que os humanos adicionam à atmosfera, o que impede que as mudanças climáticas piorem ainda mais, além de fornecer serviços ecossistêmicos essenciais para o bem-estar do planeta. O Brasil pode contribuir para mitigar as mudanças climáticas reduzindo o desmatamento da Amazônia (IPAM, 2018).

Alguns autores ressaltam que o desmatamento interfere no clima, compromete a biodiversidade e o ciclo das águas. Além disso, ele contribui para 
aprofundar os problemas sociais e não impacta positivamente o desenvolvimento econômico (FEARNSIDE, 2006; REIS; BORTOLOTTO; LOPES; BRAGA, 2017; MOURA et al., 2017)

Lovejoy e Nobre (2018, p. 1) alertam que o sistema amazônico está prestes a atingir um ponto de inflexão: "[...] We believe that negative synergies between deforestation, climate change, and widespread use of fire indicate a tipping point for the Amazon system to flip to non-forest ecosystems in eastern, southern and central Amazonia at 20-25\% deforestation"1.

De acordo com Nobre (2018) apud Alisson (2018, não paginado), “[...] Apesar de não sabermos o ponto de inflexão exato, estimamos que a Amazônia já tem $20 \%$ de área desmatada, equivalente a 1 milhão $\mathrm{km} 2$, ainda que $15 \%$ dessa área (150 mil km2) esteja em recuperação". A fim de evitar que a Amazônia atinja um limite irreversível, os pesquisadores sugerem a necessidade de não apenas controlar o desmatamento da região, mas também construir uma margem de segurança ao reduzir a área desmatada para menos de 20\% (ALISSON, 2018; LOVEJOY; NOBRE, 2018).

Nobre ressalta que é preciso zerar o desmatamento da Amazônia e o Brasil cumprir o compromisso assumido no Acordo de Paris, em 2015, de reflorestar 12 milhões de hectares de áreas desmatadas no país, das quais 50 mil km² são da Amazônia. Com essas medidas, em 2030 as áreas totalmente desmatadas na Amazônia estariam em torno de 16\% a 17\% . "Dessa forma, estaríamos no limite mais seguro, para que o desmatamento, por si só, não faça com que o bioma atinja um ponto irreversível" (ALISSON, 2018; LOVEJOY; NOBRE, 2018).

Lamentavelmente, o desmatamento na Amazônia tem aumentado, como mostra a taxa anual consolidada pelo Projeto de Monitoramento do Desmatamento na Amazônia Legal por Satélite (PRODES), do Instituto Nacional de Pesquisas Espaciais (INPE), que registrou, em 2018, $7.536 \mathrm{~km}^{2}$ e, em 2019, 10.129 km², uma variação de 34\% (INPE, 2020), ver figura 1, abaixo.

A Plataforma Intergovernamental sobre Biodiversidade e Serviços Ecossistêmicos (IPBES) divulgou um sumário alertando sobre as graves ameaças que pesam sobre a biodiversidade: um milhão de espécies de animais e vegetais no mundo estão ameaçadas de extinção. Somos um país megabiodiverso, temos responsabilidade de proteger nossa biodiversidade e nossos biomas (IPBES, 2019).

\footnotetext{
Tradução nossa: "Nós acreditamos que sinergia negativa entre desmatamento, mudança climática e uso generalizado do fogo indica um novo ponto de inflexão a partir do qual ecossistemas na Amazônia oriental, Sul e Central podem deixar de ser floresta, se o desmatamento alcançar entre $20 \%$ a $25 \%$ da floresta original".
} 
Figura 1 - Mapa do desmatamento na Amazônia Legal por quilômetro quadrado, 2018 a 2019

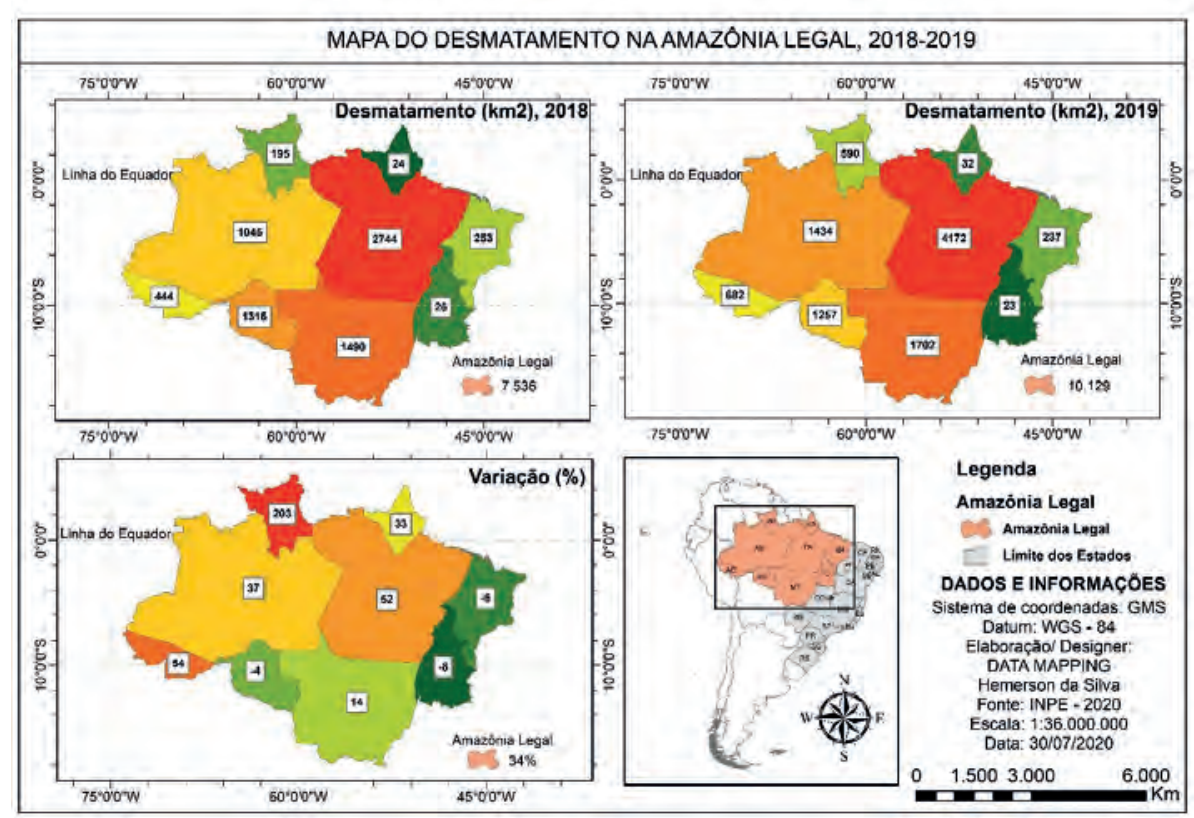

Fonte: INPE (2020).

McGrath (1997) ressalta a importância do paradigma da biodiversidade:

[...] No paradigma da biodiversidade a grande crise, que o mundo enfrenta, é a crescente taxa de perda de espécies, cuja principal causa é a destruição sem precedentes de habitat natural. [...] A floresta tropical é o ambiente mais crítico, porque a perda da floresta tropical elimina o maior número de espécies por unidade de área convertida. A bacia Amazônica, a maior extensão de floresta tropical do mundo, é a região com a maior taxa absoluta de desmatamento, é o epicentro da crise da biodiversidade (McGRATH, 1997, p. 36).

A sociedade humana tem se estruturado com base em um modelo de desenvolvimento econômico que demanda um grande consumo de energia, uso da terra e água; além do que, vivemos em um período de grandes mudanças sociais, econômicas e ecológicas, com crescimento populacional acelerado. Alguns cientistas acreditam que estamos entrando em uma nova era geológica o Antropoceno. A exploração dos recursos naturais existentes tem possibilitado este modelo de desenvolvimento. A questão é: com recursos naturais esgotados, o futuro do homem será possível neste planeta? (GROOTEN; ALMOND, 2018). 
Constatamos que diante de tão graves problemas ambientais envolvendo o planeta e a Amazônia, seria importante que o Brasil estivesse como protagonista na defesa de nosso patrimônio ambiental, entretanto vemos que o atual governo tem se empenhado em desmontar a política ambiental brasileira.

\section{DETERMINANTES AMBIENTAIS DA SAÚDE}

A ação antrópica é responsável pela mudança, até certo ponto irreversível, na diversidade da vida no planeta. A seguir, alguns indicadores da deterioração dos ecossistemas que podem afetar a saúde da população: o desmatamento, a desertificação, o estresse hídrico, os impactos na atmosfera e qualidade do ar, o impacto dos resíduos sólidos e o saneamento. Estima-se que $24 \%$ da carga mundial de morbidade e $23 \%$ de todas as mortes podem ser atribuídas a fatores relacionados com o ambiente. Nos países em desenvolvimento, a porcentagem de mortalidade atribuível a causas ambientais é de 25\% e nos desenvolvidos é de 17\% (RIOJAS-RODRÍGUEZ; ROMERO-FRANCO, 2011).

O Brasil apresenta dados preocupantes sobre saneamento. Segundo o Instituto Trata Brasil (ITB, 2017), são 33.274 .514 pessoas (16,6\%) sem acesso à água tratada e 95.775 .598 pessoas $(47,6 \%)$ sem coleta de esgoto. O Pará apresenta 6.268 .285 pessoas $(93,7 \%$ ) sem coleta de esgoto e 3.657 .011 pessoas $(54,7 \%)$ sem acesso à água tratada. A região metropolitana de Belém apresenta 1.002.666 (44,6\%) pessoas sem acesso à água tratada e 2.052.604 pessoas $(91,4 \%)$ sem coleta de esgoto.

Ressaltamos que o desmatamento impacta negativamente a saúde e tem sido associado com o aumento da exposição aos vetores da febre amarela, malária e leishmaniose (RIOJAS-RODRÍGUEZ; ROMERO-FRANCO, 2011).

Alguns autores apresentam evidências científicas da associação entre doenças e mudanças climáticas, sendo a malária a doença transmitida por vetor mais sensível às mudanças do clima (GITHEKO; LINOSAY; COFALONIERE; PATZ, 2000; OMS, 2009; PATZ; CHRISTENSON, 2011; SOUZA; AMÂNCIO; HACON; BARCELLOS, 2018).

São inúmeros os problemas de saúde decorrentes dos determinantes ambientais, por isso torna-se necessário ampliar nossa concepção do processo saúde-doença para melhor formular políticas para responder às novas demandas por saúde. Nesse sentido, problematizamos o SUS na Amazônia com novas características, preservando seus princípios, mas renovando nas abordagens. 


\section{O SUS AMAZÔNIA}

O SUS na Amazônia deve ser estruturado levando em consideração as especificidades regionais: (a) fator amazônico; (b) diversidade étnica; (c) características do território; (d) regiões de saúde; (d) novas abordagens.

O fator amazônico diz respeito a considerar maior a alocação de recursos (financeiros etc.) para a assistência à saúde na Amazônia, uma vez que a realização das atividades de saúde, muitas vezes, se dá de modo adverso dadas as longas distâncias geográficas, áreas com baixa e alta densidade demográfica e áreas com fluxo migratório.

A Amazônia tem uma rica diversidade étnica, que são os povos tradicionais: povos indígenas, ribeirinhos, quilombolas e comunidades rurais. $\mathrm{O}$ cuidado à saúde desses povos deve levar em consideração a sua cultura, seus saberes ancestrais e a situação de vulnerabilidade a que, muitas vezes, estão submetidos. Essa abordagem deve ser intermediada pela educação em saúde.

O SUS na Amazônia deve levar em consideração as características geográficas da região, no sentido de viabilizar o acesso universal à saúde: ilhas, lagos, igarapés e rios, além de considerar as pequenas localidades e os pequenos, médios e grandes municípios, pois são nestes espaços que as pessoas vivem e trabalham.

Dadas as características territoriais, é necessário reforçar as regiões com o objetivo de descentralizar as ações de saúde e facilitar o acesso das populações, por isso é preciso: (a) Implementar regiões de saúde com capacidade de planejamento, contratualização e gestão; (b) Atribuir gestão de média e alta complexidade; (c) Atribuir coordenação de vigilância à Saúde; (d) Coordenar e executar a regulação regional; (e) Instituir secretário (coordenador executivo) de saúde com equipe técnica de apoio; (f) Compor fundo orçamentário com aportes do orçamento federal, estadual e municipal para custeio e investimento (CAMPOS, 2018).

O modelo de desenvolvimento para a Amazônia tem priorizado a implantação de megahidrelétricas, a pecuária, o agronegócio e o desmatamento da floresta. Essas atividades expressam um modelo de desenvolvimento excludente, predatório, concentrador e produtor de pobreza e desigualdades.

O impacto na saúde dessas atividades (impacto de hidrelétricas, intoxicação pelo uso de agrotóxico na plantação de soja e outras culturas, a intoxicação pelo mercúrio utilizado nos garimpos de ouro, projetos de infraestrutura etc.) tem se transformado em demandas para o SUS local, que muitas vezes não consegue responder de modo eficiente. 
A nova abordagem seria o SUS reconhecer essas atividades, abordar os problemas com o conceito de impacto na saúde e mapear as seguintes áreas: (a) áreas de impacto na saúde de hidrelétricas; (b) áreas de impacto na saúde de culturas com uso de agrotóxicos; (c) áreas de impacto na saúde de garimpos e uso de mercúrio; (d) áreas de impacto na saúde de projetos de infraestrutura: rodovias, ferrovias, portos. O SUS deve se estruturar com base na especificidade de cada situação e encaminhar os problemas adequadamente para as redes de saúde e manter a vigilância à saúde.

\section{SAÚDE E BIOECONOMIA}

Segundo Vieira (2019), os ciclos produtivos que se sucederam na Amazônia em um período de quase 400 anos de história não geraram sustentabilidade ao modelo econômico adotado, o que Melo (2010) apud Vieira (2019) chamou de "progresso sem desenvolvimento":

[...] em que perduram na região a exclusão social, a pobreza, a destruição ambiental, o atraso tecnológico, a economia de enclave, a insuficiente infraestrutura logística, a fragmentação entre os setores produtivos, o nível educacional sofrível, a baixa capacidade científica instalada, as estruturas estatais ineficientes e a desigualdade social (MELO, 2010 apud VIEIRA, 2019, p. 47).

A autora ressalta ainda que temos o desafio de buscar promover a melhoria da qualidade de vida e o bem-estar da população que vive na região com equilíbrio ambiental. Nesse sentido, é fundamental pensar um modelo de desenvolvimento que coloque a cidadania, a participação democrática, socialização da política e sustentabilidade como condição de desenvolvimento nos termos analisados por Acselrad (2010, p. 21).

Com base na rica biodiversidade da floresta e saberes ancestrais, alguns autores têm defendido um modelo de desenvolvimento bioeconômico para a Amazônia, favorecendo as populações locais com geração de renda, inclusão, saúde, qualidade de vida e sustentabilidade na Amazônia (INSTITUTO HUMANITAS UNISINOS, 2019; COSTA; FERNANDES; CRISPIM, 2018).

\section{CONSIDERAÇÕES FINAIS}

A ONU alerta, neste início de século, para o colapso climático, sanitário e social decorrentes das mudanças climáticas e recomenda mudança no estilo de vida, além de proteger a água e a biodiversidade. Entretanto, o desmonte da 
política ambiental brasileira tem contribuído para aumentar o desmatamento na Amazônia, um dos determinantes das mudanças climáticas.

O desmatamento na Amazônia é desnecessário e inaceitável, entretanto, o cenário futuro é preocupante e não sinaliza para a sua redução. Alguns pesquisadores alertam que o desmatamento está chegando a um ponto de inflexão a partir do qual a Amazônia se transformaria em savana e seria irreversível.

A compreensão da dimensão ambiental da saúde que reconhece a relação saúde e ambiente e seus determinantes têm ampliado e contribuído para a explicação do processo saúde/doença. A ação antrópica sobre os ecossistemas degrada a base da vida, por isso ressaltamos que os ecossistemas são responsáveis pelos serviços ambientais e pela segurança alimentar, hídrica e climática do planeta.

O SUS deve se preparar para enfrentar os novos desafios referentes às demandas ambientais por saúde. Sugerimos pensar o SUS Amazônia com suas especificidades: fator amazônico, diversidade étnica, territorialidades, regiões de saúde e novas abordagens.

Sugerimos que o Brasil cumpra seus compromissos no Acordo de Paris, encerrando o desmatamento na Amazônia e reflorestando 12 milhões de hectares de áreas desmatadas no país, das quais 50 mil km² são na Amazônia. Apesar de o atual modelo de desenvolvimento para a Região não estar em sintonia com os pressupostos da sustentabilidade, novos projetos surgem adotando um modelo com base na biodiversidade da floresta, e nos saberes ancestrais-com perspectiva de gerar renda, inclusão, saúde e qualidade de vida para as populações amazônicas e sustentabilidade na Região.

\section{REFERÊNCIAS}

ACSERALD, H. Sustentabilidade e articulação territorial do desenvolvimento brasileiro. In: SEMINÁRIO INTERNACIONAL SOBRE DESENVOLVIMENTO REGIONAL. PROGRAMA DE PÓSGRADUAÇÃO EM DESENVOLVIMENTO REGIONAL, 2., 2010, Santa Cruz do Sul. Anais [...]. Santa Cruz do Sul: UFRGS, 2010. p. 1-47.

ALISSON, E. Desmatamento na Amazônia está prestes a atingir limite irreversível. Agência Fapesp, São Paulo, 21 fev. 2018. Disponível em: http:/ / agencia.fapesp. br/desmatamento-na-amazonia-esta-prestes-a-atingir-limite-irreversivel/27180. Acesso em: 04 jul. 2019. 
ARANDA, D. O colapso da Terra está cada vez mais próximo. Instituto Humanitas Unisinos, São Leopoldo, 08 maio 2019. Disponível em: http:// www.ihu.unisinos.br/78-noticias/588966-o-colapso-da-terra-esta-cada-vezmais-proximo. Acesso em: 19 mai. 2019.

BRASIL. Ministério do Meio Ambiente. Acordo de Paris. Ministério do Meio Ambiente, Brasília, DF, 2015. Disponível em: http://www.mma.gov.br/clima/ convencao-das-nacoes-unidas/acordo-de-paris. Acesso em: 24 jun. 2019.

CAMPOS, G. W. S. Fenômenos intervenientes no futuro do Sistema Único de Saúde-SUS. Associação Brasileira de Saúde Coletiva, Rio de Janeiro, 2018. Disponível em: https://www.abrasco.org.br/site/wp-content/ uploads / 2019/04/O-futuro-do-Sistema-\%C3\%9Anico-de-Sa\%C3\%BAde.pdf. Acesso em: 24 jun. 2019.

COSTA, F. A.; FERNANDES, D. A.; CRISPIM, C. N. S. Constituição, situação e dinâmica de Arranjos Produtivos Locais: o caso do APL de açaí na Região do Grão-Pará (2002 - 2010). Análise Econômica, Porto Alegre, v. 36, n. 69, p. 109-137, mar. 2018.

COUTO, R. C. Saúde, Problemas Ambientais e Amazônia. In: COUTO, R. C.; ACEVEDO MARÍN, R. E. (org.) Hidrelétrica Belo Monte: impactos na saúde. Belém: Amazônica Bookshelf, 2018. p. 15-46.

COUTO, R. C. S. Consolidação dos princípios do Sistema Único de Saúde. Conferência apresentada à $13^{\mathrm{a}}$ Conferência Estadual da Saúde do Pará. Belém, Pará, 13-14 de junho, 2019.

FEARNSIDE, P. Desmatamento na Amazônia: dinâmica, impactos e controle. Acta Amazônica, Manaus, v. 36, n. 3, p. 395-400, 2006.

GITHEKO, A. K.; LINOSAY, S. W.; COFALONIERE, E. U.; PATZ, J. A. Climate change and vector-borne disease: a regional analysis. Bulletin of the World Health Organization, [S. l.], v. 78, n. 9, p. 1136-1147, 2000.

GROOTEN, M.; ALMOND, R. E. A. (ed). Living Planet Report 2018: aiming higher. Switzerland: WWF, 2018. Disponível em: https://www.wwf.org.uk/sites/ default/files/2018-10/LPR2018_Full\%20Report.pdf Acesso em: 01 jul. 2019.

INPE. Monitoramento do Desmatamento da Floresta Amazônica Brasileira por Satélite. INPE, Brasília, DF, 2020. Disponível em: http://www.obt.inpe.br/ OBT/assuntos/programas/amazonia/prodes. Acesso em: 20 ago. 2020. 
INSTITUTO HUMANITAS UNISINOS. Amazônia, possível laboratório de Bioeconomia. Outras Palavras, São Paulo, 10 maio 2019. Outras mídias, descolonizações. Disponível em: https://outraspalavras.net/outrasmidias/ amazonia-possivel-laboratorio-da-bioeconomia/?fbclid=IwAR3SmPLuscrJW vz7KSYYKDCyz2F1Uv9AW_1IQ10sF8vf9A2em8LNoeoab0g. Acesso em: 13 maio 2019.

IPAM. Relatório do IPCC mostra que aumento de $2^{\circ} \mathrm{C}$ não é um limite seguro. Instituto de Pesquisa Ambiental da Amazônia, Belém, 10 out. 2018. Disponível em: https://ipam.org.br/relatorio-do-ipcc-mostra-que-aumento-de2c-no-planeta-nao-e-um-limite-seguro. Acesso em: 28 jun. 2019.

IPBES. Summary for policymakers of the global assessment report on biodiversity and ecosystem services of the Intergovernmental Science-Policy Platform on Biodiversity and Ecosystem Services, Intergovernmental Science-Policy Platform on Biodiversity and Ecosystem Services, Paris, 2019. Disponível em: https://www.ipbes.net/system/tdf/ipbes_7_10_add-1-_advance_0.pdf?file $=1 \&$ type $=$ node $\& i d=35245$. Acesso em: 19 jul. 2019.

IPCC. Global Warming of $\mathbf{1 . 5}^{\circ} \mathrm{C}$. Switzerland: IPCC, 2018. Disponível em: https://report.ipcc.ch/sr15/pdf/sr15_spm_final.pdf. Acesso em: 25 jun. 2019.

ITB. Painel de Saneamento Básico. Instituto Trata Brasil, São Paulo, 2017. Disponível em: https://www.painelsaneamento.org.br. Acesso em: 27 mai. 2019. LOVEJOY, T. E.; NOBRE, C. Amazon tipping point. Editorial. Science Advances, [S, l.], v. 4, n. 2, p. 1, Feb. 2018. Disponível em: https://advances. sciencemag.org/content/4/2/eaat2340. Acesso em: 04 jul. 2019.

McGRATH, D. G. Biosfera ou biodiversidade: uma avaliação crítica do paradigma da biodiversidade. In: XIMENES, T. (org). Perspectivas do Desenvolvimento Sustentável: uma contribuição para a Amazônia 21. Belém: UFPA/NAEA/ UNAMAZ, 1997. p. 33-69.

MILLENNIUM ECOSYSTEM ASSESSMENT. How have ecosystem change affected human well-being and poverty alleviation? In: MILLENNIUM ECOSYSTEM ASSESSMENT. Ecosystem and Human-Being: synthesis. Washington, DC: Island Press, 2005. p. 49-63. Disponível em: https:// www.millenniumassessment.org/documents/document.356.aspx.pdf. Acesso em: 01 jun. 2019. 
MOURA, R. et al. Desmatamento zero no Pará: desafios e oportunidades. Belém: Imazon, 2017. Disponível em: https://imazon.org.br/PDFimazon/ Portugues/livros/Desmatamento $\% 20$ Zero $\% 20$ no\%20Para.pdf. Acesso em: 20 fev. 2020.

OMS. Mudança climática e Saúde Humana: riscos e respostas. Sumário Revisado. Brasília, DF: OPAS, 2009.

ONU. Acordo de Paris sobre o clima. Paris: UNCC, 2015. Disponível em: https:// nacoesunidas.org/wp-content/uploads/2016/04/Acordo-de-Paris.pdf Acesso em: 24 jun. 2019.

PATZ, J.; CHRISTENSON, M. A mudança climática e a saúde. In: GALVÃO, L. A. C.; FINKELMAN, J.; HENAO, S. (org.). Determinantes ambientais e sociais da saúde. Washington, DC: OPAS, 2011. p. 215-232.

REIS, T.; BORTOLOTTO, F.; LOPES, G. R.; BRAGA, L. Desafios e oportunidades para avançar as Contribuições Nacionais no setor agropecuário e de floresta na América Latina: o caso Brasil. Belém: IPAM, 2017. Disponível em: https://ipam.org.br/wp-content/uploads/2017/04/ PCL_IPAM.pdf. Acesso em: 20 fev. 2020.

RIOJAS-RODRÍGUEZ, H.; ROMERO-FRANCO, M. A deterioração dos ecossistemas e a biodiversidade: suas implicações para a saúde humana. In: GAlvãO, L. A. C., FINKELMAN, J., HENAO, S. (org.). Determinantes ambientais e sociais da Saúde. Washington, DC: Organização Pan-Americana de Saúde; Rio de Janeiro: FIOCRUZ, 2011. p. 233-257.

SOUZA, T. C. M.; AMÂNCIO, F.; HACON, S. S.; BARCELLOS, C. Doenças sensíveis ao clima no Brasil e no Mundo: revisão sistemática. Rev. Panam. Salud Publica, [S. l.], n. 42, p. 1-10, 2018.

VIEIRA, I. C. G. Abordagens e desafios no uso de indicadores de sustentabilidade no contexto amazônico. Ciência \& Cultura, São Paulo, v. 71, n. 1, p. 46-50, jan./ mar. 2019. 